\title{
Myxomycetes Diversity of Belen Region of Hatay Province (Turkey)
}

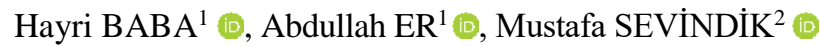 \\ ${ }^{1}$ Mustafa Kemal University, Science and Art Faculty, Biology Department, Hatay, TURKEY \\ ${ }^{2}$ Osmaniye Korkut Ata University, Bahçe Vocational High School, Osmaniye, Turkey \\ *Corresponding Author: sevindik27@gmail.com
}

Received Date: 19.07.2019

Accepted Date: 20.10.2019

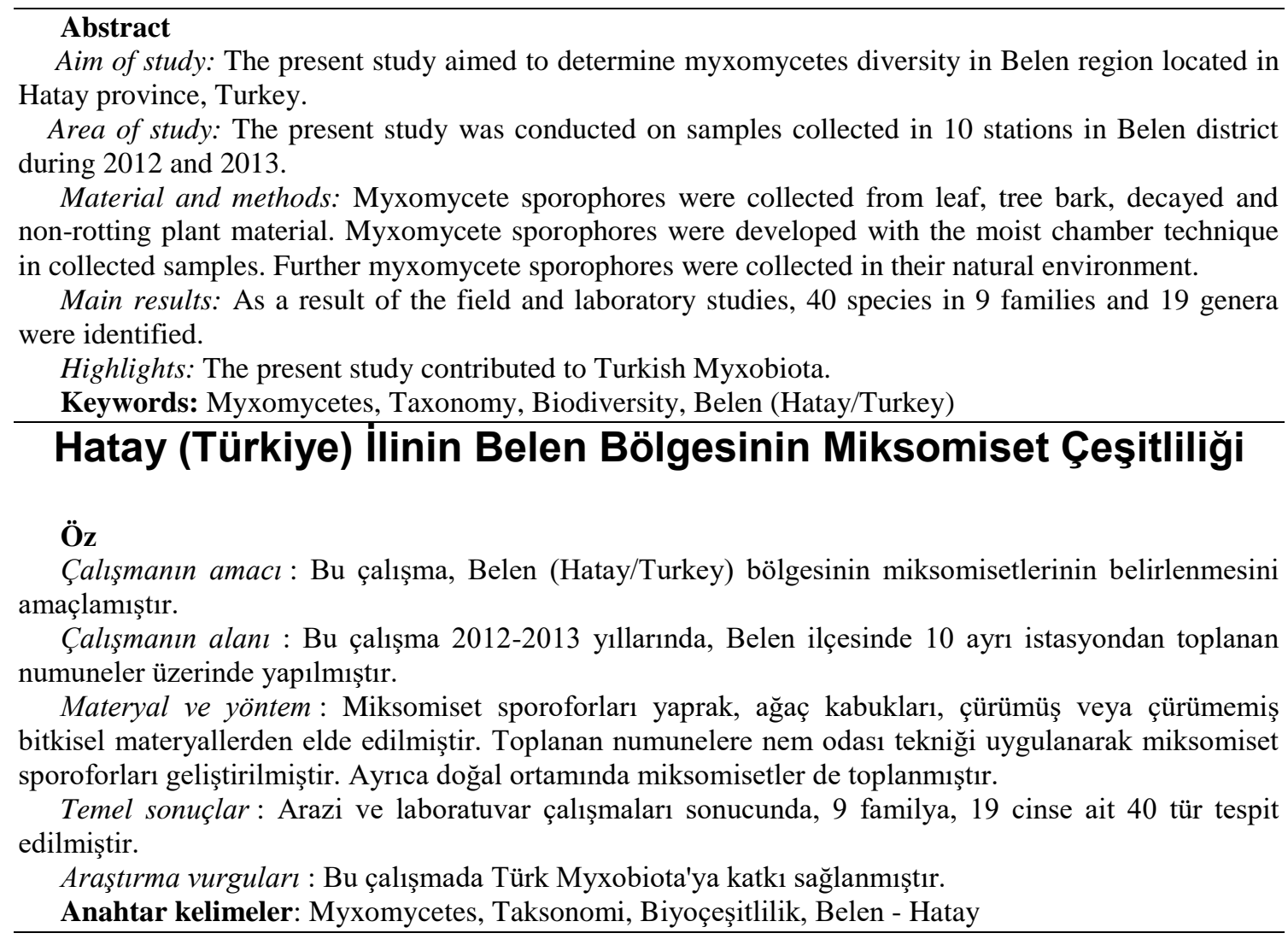

\section{Introduction}

Myxomycetes are known as true slime molds, plasmodial slime molds or Myxogastrea. Myxomycetes are multinuclear single-celled organisms that can produce one or more spores (Stephenson \& Stempen, 1994). Myxomycetes are sensitive to $\mathrm{pH}$, humidity, temperature and light, as well as the properties of the substrate on which it develops. Myxogastrea species are abundant in cool, moist and shaded areas such as rotten tree trunks, branches, alive or dead bark, decayed fruit or fruit scraps, decayed leaves and leaf debris. Myxomycetes live in the environment by feeding on other microorganisms (bacteria, yeasts, fungus hyphae, blue-green bacteria and green algae) (Farr, 1981). Myxogastrea fruit organs could develop spontaneously in the nature. Furthermore, especially after identification with the humid chamber technique, they could be detected especially on plant surfaces (Gilbert \& Martin, 1933; Härkönen \& Ukkola, 2000).

The number of known Myxomycetes is 1017 taxa globally (Lado, 2019). Two hundred and eighty-four taxa were identified in Turkey (Ergül et al., 2005a; Ergül et al., 2005b; Ergül \& Akgül, 2011; Baba et al., 2013; Süerdem et al., 2015; Baba, 2015; Baba \& Zümre, 2015; Alkan et al., 2016; Dülger et al., 2016; Ergül et al., 2016; Baba 
et al., 2016; Sesli et al., 2016; Baba \& Arslan, 2017; Baba \& Özyiğit, 2017; Baba \& Er, 2018; Baba et al., 2018 ; Ocak \& Konuk, 2018 ; Baba \& Sevindik, 2019).

The present study aimed to determine myxomycetes in different locations within the boundaries of Belen region. Also, the present study aimed to contribute to Turkish Myxobiota.

\section{Material and Methods}

Study Area

Belen is a district in Hatay province located in the Mediterranean region in Turkey. The district is surrounded by İskenderun district to the north, Antakya district to the southwest, Kirıkhan district to the east, Samandağ district to the northwest and Altınözü district to the south (Figure 1). The altitude of the district center, which is 50 $\mathrm{km}$ to Antakya, is approximately 700 meters. The total surface area of the district is 689 $\mathrm{km}^{2}$.

The elevation in the study area is between 300-1350 $\mathrm{m}$ and it can exhibit great variations within short distances. The mean annual temperature in Belen is $16.8{ }^{\circ} \mathrm{C}$. The mean annual rainfall is $697.5 \mathrm{~mm}$. The mean annual relative humidity is $65 \%$. There are pine, cedar, sycamore, larch and juniper forests between 700-1200 $\mathrm{m}$ altitudes in Amik Plain and Mediterranean sections in Belen (Anonymous, 2019).

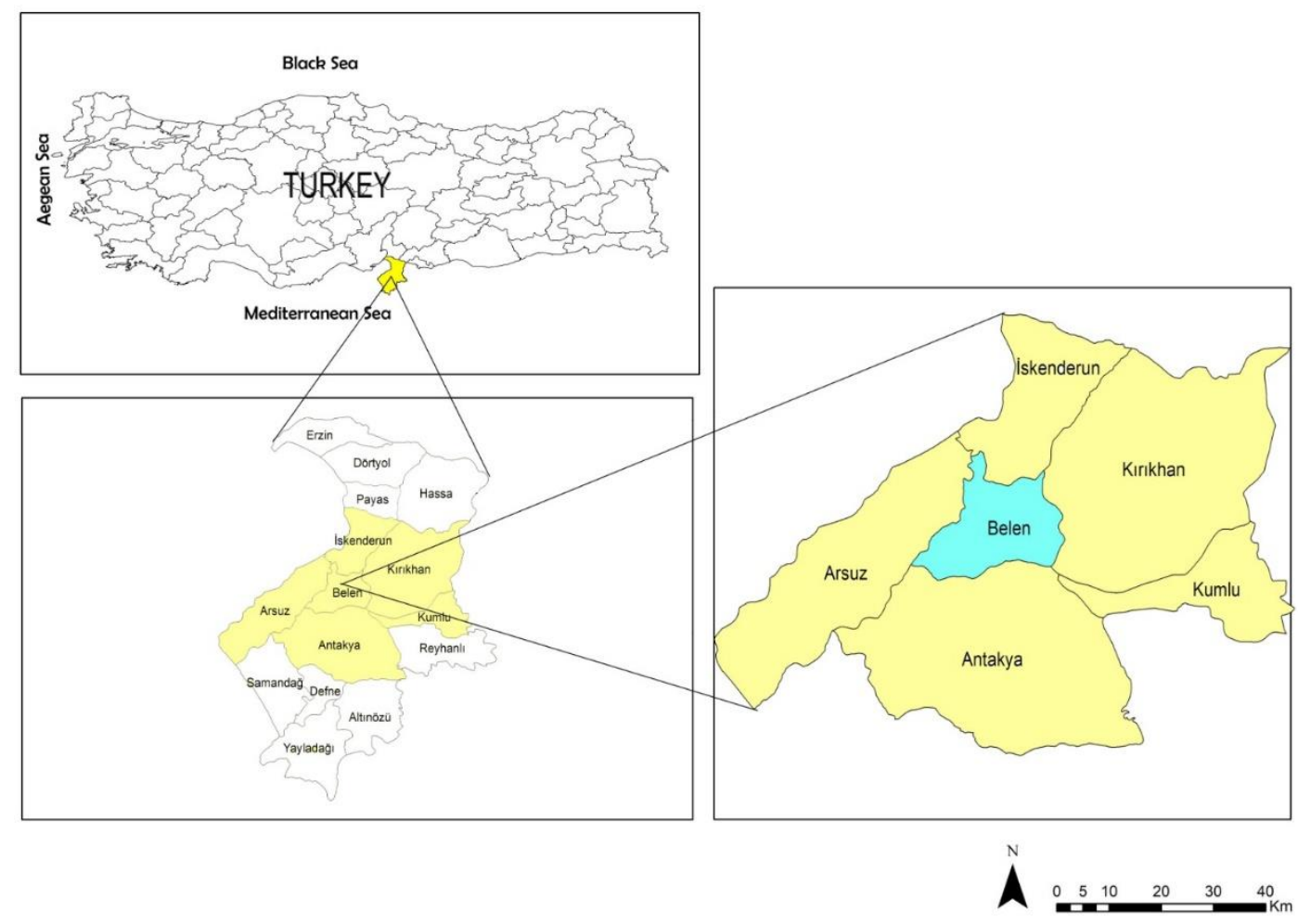

Figure 1. Map of the research area

\section{Samplings and Identifications}

In the field studies, myxomycetes fruits that were collected in different regions of Belen district were transferred to the laboratory in small carton boxes.

Material that did not contain myxomycetes and sporophore samples but that contained myxomycet spores (tree bark, cut tree stumps, rash and decaying leaves, pointer, cones, fruit and residues of vegetable material) were placed in small zip-lock storage bags and transferred to the laboratory. Then, in the laboratory, it was ensured that they were able to form fructification with Moist Chamber Technique developed by Gilbert \& Martin (1933). 


\section{Moist Chamber Technique}

A double layer of sterile filter paper was placed in petri dishes or transparent storage containers. Samples were then placed in these containers and distilled water was added. The samples were then allowed to swell for 24-48 hours. The samples were monitored with light stereomicroscope at regular intervals. The collected samples were prepared by placing one or two layers of blotting paper in petri dishes inside the storage containers and the samples were allowed to dry at room temperature. Then, the samples were turned into fungaryum material (Gilbert \& Martin, 1933 ; Härkönen \& Ukkola, 2000).

The general structure, shape, color, macroscopic measurements, capillitium, presence of pseudocapillitium and columella, if any, shape measurements, shape, color, size and spore ornamentations of the spores were investigated in detail. Samples were identified based on the literature (Martin \& Alexopoulos, 1969; Farr, 1981; Thind, 1977; Martin, et al., 1983; Neubert et al., 1993; 1995; 2000; Stephenson \& Stempen, 1994; Lado \& Pando, 1997; Ing, 1999; Sesli et al., 2016). The fungarium material of the identified samples were stored in the Department of Biology Laboratory at MKU Faculty of Science and Arts.

\section{Results}

The present study was the first systematic study on myxomycetes in Belen region. In the present study conducted in the district of Belen (Hatay) in 2012-2013, 561 sporophores were collected in different locations and processed in the laboratory. A total of 303 myxomycete sporophores were obtained from these sporophores. Fourtythree sporophores were collected from the nature, and 260 sporophores were obtained with the moist chamber culture. As a result of the identification of myxomycete sporophores obtained from natural environment and moist chamber culture, 40 species were identified in 5 ordo, 9 families and 19 genera.

The taxa were listed alphabetically. The habitat, settlement, collection date, altitude, geographical location, sample numbers of the sporophores are indicated.

\section{Systematic classification}

Eukaryota

Protozoa

Amoebozoa

Myxomycetes

\section{Ceratiomyxales}

1. Ceratiomyxa fruticulosa (O.F. Müll.) T. Macbr., Kömürçukuru, on Pinus sp. wood, Er. 382, 28.04.2013 569 m. $35^{\circ} 53^{\prime} 20^{\prime \prime} \mathrm{N}$; $36^{\circ} 05^{\prime} 28^{\prime \prime} \mathrm{E}$.

\section{Echinosteliales}

Echinosteliaceae

2. Echinostelium minutum de Bary, Atik, on Pinus brutia Ten. wood, Er. 222, 24.12.2012 $50735^{\circ} 54^{\prime} 45^{\prime \prime} \mathrm{N} ; 36^{\circ} 02^{\prime} 14 " \mathrm{E}$; Osman Tiryaki Forest, on P. brutia wood, Er. 13, 18.11.2012 $501 \mathrm{~m} .35^{\circ} 54^{\prime} 39^{\prime \prime} \mathrm{N} ; 36^{\circ} 00^{\prime} 55^{\prime \prime}$ E; Güzelyayla, on the rash, Er. 333,

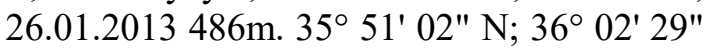
E; Akgün, on P. brutia wood, Er. 174, 15.12.2012 489m. $35^{\circ} 54^{\prime} 21^{\prime \prime} \mathrm{N} ; 36^{\circ} 01^{\prime} 41^{\prime \prime}$ E; Gedik, on the rash, Er. 183, 25.11.2012 $516 \mathrm{~m}$. $35^{\circ} 54^{\prime} 13^{\prime \prime} \mathrm{N} ; 36^{\circ} 01^{\prime} 00^{\prime \prime} \mathrm{E}$.

\section{Liceales \\ Cribrariaceae}

3. Cribraria cancellata (Batsch) Nann.Bremek., Kömürçukuru, on P. brutia wood, Er. 384, 28.04.2013 569m. 35 53' 20" N; $36^{\circ} 05^{\prime} 28^{\prime \prime}$ E; Müftüler, on $P$. brutia wood, Er. 529, 14.04.2013 589m. 35 53' 24" N; $36^{\circ} 04^{\prime} 50^{\prime \prime}$ E; Gedik, on P. brutia wood, Er. $125,25.11 .2012516 \mathrm{~m} .35^{\circ} 54^{\prime} 13^{\prime \prime} \mathrm{N} ; 36^{\circ}$ 01' 00" E.

4. C. violacea Rex., Akgün, on Pinus sp. wood, Er. 163, 15.12.2012 489m. 35 54' 21" $\mathrm{N} ; 36^{\circ} 01^{\prime}$ 41" E; Osman Erdal Tiryaki Forest, on Quercus sp. wood, Er. 18, 18.11.2012 $501 \mathrm{~m} .35^{\circ} 54^{\prime} 39^{\prime \prime} \mathrm{N} ; 36^{\circ} 00^{\prime} 55^{\prime \prime}$ E; Atik, on Pinus sp. wood, Er. 228, 24.12.2012 507m. 35० 54' 45" N; 36 02' 14" E; Güzelyayla, on Pinus sp. wood, Er. 335, 26.01.2013 486m. 35 51' 02" N; 36 02' 29" E; Şenbük, on Pinus sp. wood, Er. 298, 26.01.2013 460m. $35^{\circ} 54^{\prime} 21^{\prime \prime} \mathrm{N} ; 36^{\circ} 01^{\prime} 41^{\prime \prime}$ E.

\section{Liceaceae}

5. Licea castanea G. Lister, Kömürçukuru, on P. brutia wood, Doğal, Er. 386, 28.04.2013 569m. $35^{\circ} 53^{\prime} 20^{\prime \prime} \mathrm{N} ; 36^{\circ} 05^{\prime} 28^{\prime \prime}$ 
E; Osman Erdal Tiryaki Forest, on P. brutia wood, Er. 88, 18.11 .2012501 m. 35 54' 39" N; $36^{\circ} 00^{\prime} 55^{\prime \prime}$ E; Müftüler, on Pinus sp., Er. $533,14.04 .2013589 \mathrm{~m}$. $35^{\circ} 53^{\prime} 24^{\prime \prime} \mathrm{N} ; 36^{\circ}$ 04 ' 50" E.

6. L. kleistobolus G.W. Martin, Atik, on $P$. brutia wood, Er, 223, 24.12.2012 507m. 35 ${ }^{\circ}$ $54^{\prime} 45^{\prime \prime} \mathrm{N} ; 36^{\circ} 02^{\prime} 14^{\prime \prime} \mathrm{E}$; Güzelyayla, on $P$. brutia wood, Er. $338,26.01 .2013486 \mathrm{~m}$. $35^{\circ}$ $51^{\prime} 02^{\prime \prime} \mathrm{N} ; 36^{\circ} 02^{\prime} 29^{\prime \prime} \mathrm{E}$; Gedik, on P. brutia wood, Er. 190, 25.11.2012 516m. 35 54' 13" $\mathrm{N} ; 36^{\circ} 01^{\prime} 00^{\prime \prime} \mathrm{E}$

7. L. minima Fr., Güzelyayla, on $P$. brutia wood, Er. 338, 26.01.2013 486m. 35 51' 02" $\mathrm{N} ; 36^{\circ} 02^{\prime} 29^{\prime \prime} \mathrm{E}$; Atik, on P. brutia wood, Er. $24124.12 .2012507 \mathrm{~m} .35^{\circ} 54^{\prime} 45^{\prime \prime} \mathrm{N} ; 36^{\circ}$ 02' 14" E.

\section{Trichiales}

\section{Arcyriaceae}

8. Arcyria cinerea (Bull.) Pers., Osman Erdal Tiryaki forest, on Pinus brutia Ten. wood, Er. 13, $18.11 .2012501 \mathrm{~m} .35^{\circ} 54^{\prime} 39^{\prime \prime} \mathrm{N} ; 36^{\circ}$ 00' 55" E; on Pinus the bark, Er. 37, $18.11 .2012501 \mathrm{~m} .35^{\circ} 54^{\prime} 39^{\prime \prime} \mathrm{N} ; 36^{\circ} 00^{\prime} 55^{\prime \prime}$ $\mathrm{E}$; on the rash, Er. $59,18.11 .2012501 \mathrm{~m} .35^{\circ}$ $54^{\prime} 39^{\prime \prime} \mathrm{N} ; 36^{\circ} 00^{\prime} 55^{\prime \prime} \mathrm{E}$; Gedik, on P. brutia wood, Er. 109, 25.11.2012 516m. 35 54' $13 "$ N; 36 01' 00" E; on Pinus the bark, Er. $106,25.11 .2012516 \mathrm{~m} .35^{\circ} 54^{\prime} 13^{\prime \prime} \mathrm{N} ; 36^{\circ}$ 01' 00" E; Akgün, on P. brutia wood, Er. $147,15.12 .2012489 \mathrm{~m} .35^{\circ} 54^{\prime} 21^{\prime \prime} \mathrm{N} ; 36^{\circ}$ 01' 41" E; on Pinus the bark, Er. 148, $15.12 .2012489 \mathrm{~m}$. $35^{\circ} 54^{\prime} 21^{\prime \prime} \mathrm{N} ; 36^{\circ} 01^{\prime} 41^{\prime \prime}$ $\mathrm{E}$; on the rash, Er. 159, 15.12.2012 $489 \mathrm{~m}$. $35^{\circ} 54^{\prime} 21^{\prime \prime} \mathrm{N} ; 36^{\circ} 01^{\prime} 41^{\prime \prime} \mathrm{E}$; Atik, on $P$. brutia wood, Er. 224, $24.12 .2012507 \mathrm{~m} .35^{\circ}$ $54^{\prime} 45^{\prime \prime} \mathrm{N} ; 36^{\circ} 02^{\prime} 14^{\prime \prime} \mathrm{E}$, on the rash, Er. 224 ,

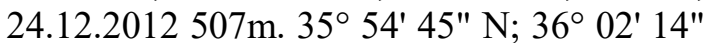
E; Senbük, on P. brutia wood, Er. 293, 26.01.2013 460m. 35० 54' 21" N; 360 01' 41" E; Güzelyayla, on P. brutia wood, Er. 333,

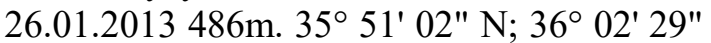
E; Kömürçukuru, on P. brutia wood, Er. $383,28.04 .2013569 \mathrm{~m}$. $35^{\circ} 53^{\prime} 20^{\prime \prime} \mathrm{N} ; 36^{\circ}$ 05' 28" E; on P. brutia wood, Er. 380, $28.04 .2013569 \mathrm{~m}$. $35^{\circ} 53^{\prime} 20^{\prime \prime} \mathrm{N} ; 36^{\circ} 05^{\prime} 28^{\prime \prime}$ E; Müftüler, on P. brutia wood, Er. 514, $14.04 .2013589 \mathrm{~m}$. $35^{\circ} 53^{\prime} 24^{\prime \prime} \mathrm{N} ; 36^{\circ} 04^{\prime} 50^{\prime \prime}$ E; Soğukoluk, on Pinus sp. wood, Er. 472, 24.02.2013 585m. 35 53' 15" N; 360 06' 36" E; K1c1, on P. brutia wood, Er. 508,
12.02.2013 540m. $35^{\circ} 53^{\prime} 23^{\prime \prime} \mathrm{N} ; 36^{\circ} 03^{\prime} 54^{\prime \prime}$ E.

9. A. globosa Schwein, Akgün, on Pinus sp. wood, Er. $148,15.12 .2012489 \mathrm{~m} .35^{\circ} 54^{\prime} 21^{\prime \prime}$ N; 360 01' 41" E; Güzelyayla, on Quercus sp. wood, Er. 332, 26.01.2013 486m. 35० 51' 02" $\mathrm{N} ; 36^{\circ} 02^{\prime} 29^{\prime \prime} \mathrm{E}$;

10. A. incarnata (Pers. ex J.F. Gmel.) Pers., Osman Erdal Tiryaki Forest, on P. brutia bark and wood, Er. 22, 18.11.2012 $501 \mathrm{~m}$. $35^{\circ} 54^{\prime} 39^{\prime \prime} \mathrm{N}$; $36^{\circ} 00^{\prime} 55^{\prime \prime} \mathrm{E}$; Akgün, on $P$. brutia the bark and wood, Er. 163, 15.12 .2012489 m. $35^{\circ} 54^{\prime} 21^{\prime \prime} \mathrm{N} ; 36^{\circ} 01^{\prime} 41^{\prime \prime}$ E; Atik, on P. brutia wood, Er. 224, 24.12.2012 507 m. $35^{\circ} 54^{\prime} 45^{\prime \prime} \mathrm{N} ; 36^{\circ} 02^{\prime} 14^{\prime \prime}$ E; Gedik, on P. brutia wood, Er. 117, 25.11.2012 516 m. $35^{\circ} 54^{\prime} 13^{\prime \prime} \mathrm{N} ; 36^{\circ} 01^{\prime} 00^{\prime \prime}$ E; Güzelyayla, on Pinus sp. wood, Er. 333, 26.01.2013 $486 \mathrm{~m}$. $35^{\circ} 51^{\prime} 02^{\prime \prime} \mathrm{N} ; 36^{\circ} 02^{\prime} 29^{\prime \prime}$ E.

11. A. minuta Buchet, Atik, on Quercus sp. wood, Baba. 234, 24.12.2012 507m. 35 54' $45^{\prime \prime} \mathrm{N} ; 36^{\circ} 02^{\prime} 14^{\prime \prime} \mathrm{E}$.

12. A. obvelata (Oeder) Onsberg, Gedik, on Pinus sp. wood, Baba. 121, 25.11.2012 $516 \mathrm{~m}$. $35^{\circ} 54^{\prime} 13^{\prime \prime} \mathrm{N} ; 36^{\circ} 01^{\prime}$ 00" E; Atik, on

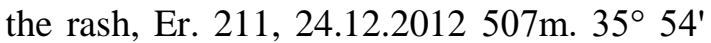
$45^{\prime \prime} \mathrm{N} ; 36^{\circ} 02^{\prime} 14 "$ E; Osman Erdal Tiryaki Forest, on the rash, Er. 3, 18.11.2012 $501 \mathrm{~m}$. $35^{\circ} 54^{\prime} 39^{\prime \prime} \mathrm{N}$; $36^{\circ} 00^{\prime} 55^{\prime \prime} \mathrm{E}$;

13. A. pomiformis (Leers) Rostaf., Osman Erdal Tiryaki Forest, on P. brutia wood, Baba. 59, 18.11.2012 501 m. 35 54' 39" N; $36^{\circ} 00^{\prime} 55^{\prime \prime} \mathrm{E}$; Akgün, on P. brutia bark and wood, Er. 160, 15.12.2012 489m. 35 54' 21" $\mathrm{N} ; 36^{\circ} 01^{\prime} 41^{\prime \prime} \mathrm{E}$; Atik, on P. brutia wood, Er. 222, 24.12.2012 507m. $35^{\circ} 54^{\prime} 45^{\prime \prime} \mathrm{N}$; $36^{\circ} 02^{\prime} 14^{\prime \prime} \mathrm{E}$; Soğukoluk, on P. brutia wood, Er. 467, 24.02.2013 585m. 35 53' 15" $\mathrm{N} ; 36^{\circ} 06^{\prime} 36^{\prime \prime} \mathrm{E}$; Gedik, on P. brutia wood, Er. 110, 25.11.2012 516m. 35 54' 13" N; $36^{\circ} 01^{\prime}$ 00" E; Güzelyayla, on Pinus sp. wood, Er. 295, 26.01.2013 486m. 35 51' 02" N; 3602' 29" E; Kömürçukuru, on Pinus sp. wood, Er. 389, 28.04.2013 569m. 35 53' 20" $\mathrm{N} ; 36^{\circ} 05^{\prime} 28^{\prime \prime} \mathrm{E}$; Müftüler, on $P$. brutia wood, Er. 515, 14.04.2013 589m. 35 53' 24" $\mathrm{N} ; 36^{\circ} 04^{\prime} 50^{\prime \prime} \mathrm{E}$.

\section{Trichiaceae}

14. Hemitrichia sp. Rostaf., Atik, on $P$. brutia wood, Er. 229, 24.12.2012 507m. 35 ${ }^{\circ}$ $54^{\prime} 45^{\prime \prime} \mathrm{N}$; $36^{\circ} 02^{\prime} 14^{\prime \prime} \mathrm{E}$; Şenbük, on $P$. 
brutia wood, Er, 297, 26.01.2013 460m. 35 $54^{\prime} 21^{\prime \prime} \mathrm{N} ; 36^{\circ} 01^{\prime} 41^{\prime \prime} \mathrm{E}$.

15. Trichia decipiens (Pers.) T. Macbr., N. Amer, Atik, on the rash, Er. 221, 24.12.2012 $507 \mathrm{~m}$. $35^{\circ} \quad 54^{\prime} \quad 45^{\prime \prime} \mathrm{N} ; 36^{\circ} 02^{\prime} \quad 14^{\prime \prime} \quad \mathrm{E}$; Müftüler, on the rash, Er. 510, 14.04.2013 $589 \mathrm{~m}$. 35 $55^{\circ}$ ' 24" N; 360 04' 50" E; Şenbük, on P. brutia wood, Er. 297, 26.01.2013 $460 \mathrm{~m} .35^{\circ} 54^{\prime} 21^{\prime \prime} \mathrm{N} ; 36^{\circ} 01^{\prime} 41^{\prime \prime} \mathrm{E}$.

16. T. munda (Lister) Meyl., Akgün, on $P$. brutia wood, Er. 53, 15.12.2012 489m. $35^{\circ}$ $54^{\prime} 21^{\prime \prime} \mathrm{N} ; 36^{\circ} 01^{\prime} 41^{\prime \prime}$ E; Gedik, on P. brutia wood, Er. 112, 25.11.2012 516m. 35० 54' 13" $\mathrm{N} ; 36^{\circ} 01^{\prime} 00^{\prime \prime}$ E; Kömürçukuru, on $P$. brutia wood, Er. 384, 28.04.2013 569m. 35० 53' 20" $\mathrm{N} ; 36^{\circ} 05^{\prime} 28^{\prime \prime} \mathrm{E}$

\section{Physarales}

Didymiaceae

17. Diderma hemisphaericum (Bull.) Hormen., Güzelyayla, on $P$. brutia wood, Baba. 338, 26.01.2013 486m. 35 51' 02" N; $36^{\circ} 02^{\prime} 29^{\prime \prime} \mathrm{E}$.

18. Didymium clavus (Alb. \& Schwein.) Rabenh., Atik, on P. brutia wood, Baba. 234,

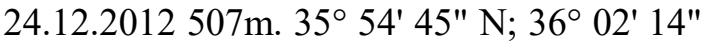
E.

19. D. megalosporum Berk. \& M.A. Curtis, Kömürçukuru, on Pinus sp. wood, Er 487,

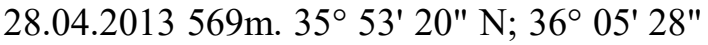
E.

20. D. squamulosum (Alb.\& Schw.) Fries, Atik, on Pinus the bark, Er. 265, 24.12.2012 $507 \mathrm{~m}$. $35^{\circ} 54^{\prime} 45^{\prime \prime} \mathrm{N} ; 36^{\circ} 02^{\prime} 14^{\prime \prime} \mathrm{E}$.

\section{Physaraceae}

21. Badhamia dubia Nann.-Bremek., Atik, on Quercus sp. bark, Er. 223, 24.12.2012 $507 \mathrm{~m}$. $35^{\circ} 54^{\prime} 45^{\prime \prime} \mathrm{N} ; 36^{\circ} 02^{\prime} 14^{\prime \prime} \mathrm{E}$.

22. B. panicea (Fries) Rost., Atik, on Quercus sp. bark, Er. 228. 24.12.2012 507m. $35^{\circ} 54^{\prime} 45^{\prime \prime} \mathrm{N} ; 36^{\circ} 02^{\prime} 14^{\prime \prime} \mathrm{E}$.

23. Craterium sp. Trentep., Kömürçukuru, on P. brutia leaf, Baba. 385, 28.04.2013 $569 \mathrm{~m}$. $35^{\circ} 53^{\prime} 20^{\prime \prime} \mathrm{N} ; 36^{\circ} 05^{\prime} 28^{\prime \prime} \mathrm{E}$;

24. C. dictyosporum (Rostaf.) H. Neubert, Nowotny \& K. Baumann, Akgün, on $P$. brutia wood, Baba. 148, 15.12.2012 489m. $35^{\circ} 54^{\prime} 21^{\prime \prime} \mathrm{N} ; 36^{\circ} 01^{\prime} 41^{\prime \prime}$ E; Kömürçukuru, on P. brutia wood, Er. 384, 28.04.2013 $569 \mathrm{~m} .35^{\circ} 53^{\prime} 20^{\prime \prime} \mathrm{N} ; 36^{\circ} 05^{\prime} 28^{\prime \prime} \mathrm{E}$;.

25. Physarum sp. Pers., Osman Erdal Tiryaki Forest, on Pinus sp. wood, Baba. 7,
18.11.2012 $501 \mathrm{~m} .35^{\circ} 54^{\prime} 39^{\prime \prime} \mathrm{N} ; 36^{\circ} 00^{\prime} 55^{\prime \prime}$ E.

26. P. album (Bull.) Chevall., Osman Erdal Tiryaki Forest, on $P$. brutia wood, Er. 9, 18.11.2012 $501 \mathrm{~m}$. $35^{\circ} 54^{\prime} 39^{\prime \prime} \mathrm{N} ; 36^{\circ} 00^{\prime} 55^{\prime \prime}$ E; Akgün, on P. brutia wood, Er. 148, 15.12.2012 489m. 35 $54^{\prime} 21^{\prime \prime} \mathrm{N} ; 36^{\circ} 01^{\prime} 41^{\prime \prime}$ E; Kömürçukuru, on P. brutia wood, Er. 385 , 28.04.2013 569m. $35^{\circ} 53^{\prime} 20^{\prime \prime} \mathrm{N} ; 36^{\circ} 05^{\prime} 28^{\prime \prime}$ $\mathrm{E}$;.

27. P. oblatum Macbr., Osman Erdal Tiryaki Forest, on Pinus sp. wood, Er. 36, $18.11 .2012501 \mathrm{~m} .35^{\circ} 54^{\prime} 39^{\prime \prime} \mathrm{N} ; 36^{\circ} 00^{\prime} 55^{\prime \prime}$ E.

28. P. robustum (Lister) Nann -Bremek, Atik, on Pinus the bark, Baba. 225, 24.12.2012 507m. $35^{\circ} 54^{\prime} 45^{\prime \prime} \mathrm{N} ; 36^{\circ} 02^{\prime} 14^{\prime \prime}$ E.

\section{Stemonitales \\ Stemonitidaceae}

29. Collaria lurida (G. Lister) Nann.Bremek., Osman Erdal Tiryaki Forest, on Pinus sp. wood, Er. 6, 18.11.2012 501 m. 35 $54^{\prime} 39^{\prime \prime}$ N; 36 00' 55" E; Gedik, on Pinus sp. wood, Er 122; 25.11.2012 516m. 35 54' 13" N; $36^{\circ} 01^{\prime} 00^{\prime \prime}$ E; Akgün, on Pinus sp. wood,

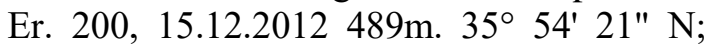
$36^{\circ} 01^{\prime} 41^{\prime \prime}$ E; Atik, on Pinus sp. wood, Er. $256,24.12 .2012507 \mathrm{~m}$. $35^{\circ} 54^{\prime} 45^{\prime \prime} \mathrm{N} ; 36^{\circ}$ 02' 14" E.

30. Comatricha ellae Härk., Osman Erdal Tiryaki Forest, on P. brutia wood, Er. 11, 18.11.2012 $501 \mathrm{~m} .35^{\circ} 54^{\prime} 39^{\prime \prime} \mathrm{N} ; 36^{\circ} 00^{\prime} 55^{\prime \prime}$ E; Gedik, on P. brutia wood, Baba. 113, 25.11.2012 516m. 35 $54^{\prime} 13^{\prime \prime} \mathrm{N} ; 36^{\circ} 01^{\prime} 00^{\prime \prime}$ E; Akgün, on P. brutia wood, Baba. 167, 15.12.2012 489m. 35० 54' 21" N; 36 01' 41" E; Atik, on P. brutia wood, Baba. 261, 24.12.2012 507m. 35० 54' 45" N; 360 02' 14" E; Şenbük, on $P$. brutia wood, Er. 296, 26.01.2013 460m. 35० 54' 21" N; 360 01' 41" E; Güzelyayla, on P. brutia wood, Er. 335, 26.01.2013 486m. 35० 51' 02" N; 360 02' 29" E; Kömürçukuru, on P. brutia wood, Er. 377, $28.04 .2013569 \mathrm{~m}$. $35^{\circ} 53^{\prime} 20^{\prime \prime} \mathrm{N} ; 36^{\circ} 05^{\prime} 28^{\prime \prime}$ E; Soğukoluk, on P. brutia wood, Er. 460, 24.02.2013 585m. $35^{\circ} 53^{\prime} 15^{\prime \prime} \mathrm{N} ; 36^{\circ} 06^{\prime} 36^{\prime \prime}$ E.

31. Comatricha nigra (Pers.) J. Schröt., Atik, on P. brutia wood and on Quercus sp. wood, Er. 222, 24.12.2012 507m. $35^{\circ} 54^{\prime} 45^{\prime \prime} \mathrm{N}$; $36^{\circ} 02^{\prime} 14 "$ E; Osman Erdal Tiryaki Forest, 
on P. brutia wood, Baba. 5, 18.11.2012 501 m. $35^{\circ} 54^{\prime} 39^{\prime \prime} \mathrm{N}$; $36^{\circ} 00^{\prime} 55^{\prime \prime} \mathrm{E}$; Akgün, on P. brutia wood, Er. 113, 15.12.2012 489m. $35^{\circ} 54^{\prime} 21^{\prime \prime} \mathrm{N} ; 36^{\circ} 01^{\prime} 41^{\prime \prime}$ E; Gedik, on $P$. brutia wood, Baba. 181, 25.11.2012 516m. $35^{\circ} 54^{\prime} 13^{\prime \prime}$ N; $36^{\circ} 01^{\prime} 00^{\prime \prime}$ E; Şenbük, on $P$. brutia wood, Er. 294, $26.01 .2013460 \mathrm{~m} .35^{\circ}$ 54' 21" N; 36 01' 41" E; Güzelyayla, on $P$. brutia wood, Er. 338, 26.01.2013 486m. 35 51' 02" N; 36 02' 29" E; Kömürçukuru, on P. brutia wood, Er. 373, 28.04.2013 569m. $35^{\circ} 53^{\prime} 20^{\prime \prime} \mathrm{N} ; 36^{\circ} 05^{\prime} 28^{\prime \prime}$ E.

32. Enerthenema papillatum (Pers.) Rostaf, Gedik, on P. brutia Ten. wood, Er. 106, 25.11.2012 516m. 35० 54' 13" N; 360 01' 00" E; Akgün, on P. brutia wood, Baba, 148, 15.12.2012 489m. 35० 54' 21" N; 36 01' 41" E; Atik, on P. brutia wood, Er, 241, 24.12.2012 507m. $35^{\circ} 54^{\prime} 45^{\prime \prime} \mathrm{N} ; 36^{\circ} 02^{\prime} 14^{\prime \prime}$ E.

33. Lamproderma arcyrioides (Sommerf.) Rostaf., Güzelyayla, on P. brutia Ten. Er. $333,26.01 .2013486 \mathrm{~m}$. $35^{\circ} 51^{\prime} 02^{\prime \prime} \mathrm{N} ; 36^{\circ}$ $02^{\prime} 29^{\prime \prime}$ E; Soğukoluk, on P. brutia wood, Er. 472, $24.02 .2013585 \mathrm{~m}$. $35^{\circ} 53^{\prime} 15^{\prime \prime} \mathrm{N} ; 36^{\circ}$ $06 ' 36^{\prime \prime}$ E; K1c1, on P. brutia wood, Er. 506, $12.02 .2013540 \mathrm{~m}$. $35^{\circ} 53^{\prime} 23^{\prime \prime} \mathrm{N} ; 36^{\circ} 03^{\prime} 54^{\prime \prime}$ E.

34. Macbrideola cornea (G. Lister \& Cran) Alexop., Akgün, on Pinus sp. wood, Baba. $166,15.12 .2012489 \mathrm{~m} .35^{\circ} 54^{\prime} 21^{\prime \prime} \mathrm{N} ; 36^{\circ}$ 01 ' 41 " E.

35. M. decapillata H. C. Gilbert, Şenbük, on Pinus sp. wood, Baba, 297, 26.01.2013 $460 \mathrm{~m} .35^{\circ} 54^{\prime} 21^{\prime \prime} \mathrm{N} ; 36^{\circ} 01^{\prime} 41^{\prime \prime} \mathrm{E}$.
36. Stemonitis fusca Roth., Gedik, on $P$. brutia wood, Baba. 127, 25.11.2012 516m. $35^{\circ} 54^{\prime} 13^{\prime \prime} \mathrm{N} ; 36^{\circ} 01^{\prime} 00^{\prime \prime} \mathrm{E}$.

37. Stemonitopsis amoena (Nann.-Bremek.) Nann.-Bremek., Osman Erdal Tiryaki Forest, on P. brutia wood, Er. 36, 18.11.2012 $501 \mathrm{~m}$. $35^{\circ} 54^{\prime} 39^{\prime \prime} \mathrm{N} ; 36^{\circ} 00^{\prime} 55^{\prime \prime} \mathrm{E}$; Gedik, on $P$. brutia wood, Er. 117, 25.11.2012 516m. 35 $54^{\prime} 13^{\prime \prime} \mathrm{N} ; 36^{\circ} 01^{\prime} 00^{\prime \prime} \mathrm{E}$; Akgün, on P. brutia wood, Er. 156, 15.12.2012 489m. 35 54' 21" $\mathrm{N} ; 36^{\circ} 01^{\prime} 41^{\prime \prime} \mathrm{E}$; Soğukoluk, on P. brutia wood, Er. 469, 24.02.2013 585m. 35 53' 15" $\mathrm{N} ; 36^{\circ} 06^{\prime} 36^{\prime \prime} \mathrm{E}$.

38. S. hyperopta (Meylan) Nann.-Bremek., Akgün, on $P$. brutia wood, Baba. 169, 15.12.2012 489m. $35^{\circ} 54^{\prime} 21^{\prime \prime} \mathrm{N} ; 36^{\circ} 01^{\prime} 41^{\prime \prime}$ E.

39. S. reticulata (H.C.Gilbert) Nann.Bremek., Atik, on P. brutia wood, Er. 230, 24.12.2012 507m. $35^{\circ} 54^{\prime} 45^{\prime \prime} \mathrm{N} ; 36^{\circ} 02^{\prime} 14^{\prime \prime}$ E; Gedik, on P. brutia wood, Er. 118, 25.11.2012 516m. $35^{\circ} 54^{\prime} 13^{\prime \prime} \mathrm{N} ; 36^{\circ} 01^{\prime} 00^{\prime \prime}$ E.

40. S. subcaespitosa (Peck) Nann.-Bremek., Osman Erdal Tiryaki Forest, on P. brutia wood, Baba. 4, 18.11 .2012501 m. 35 54' $39^{\prime \prime} \mathrm{N} ; 36^{\circ} 00^{\prime} 55^{\prime \prime} \mathrm{E}$; Akgün, on P. brutia wood, Er. 157, 15.12.2012 489m. 35 54' 21" $\mathrm{N}$; $36^{\circ} 01^{\prime} 41^{\prime \prime}$ E; Kömürçukuru, on $P$. brutia wood, Er. 383, 28.04.2013 569m. 35 53' 20" $\mathrm{N}$; $36^{\circ} 05^{\prime} 28^{\prime \prime}$ E; Güzelyayla, on P. brutia wood, Er. 335, 26.01.2013 486m. 35 51' 02" $\mathrm{N} ; 36^{\circ} 02^{\prime} 29^{\prime \prime} \mathrm{E}$. 


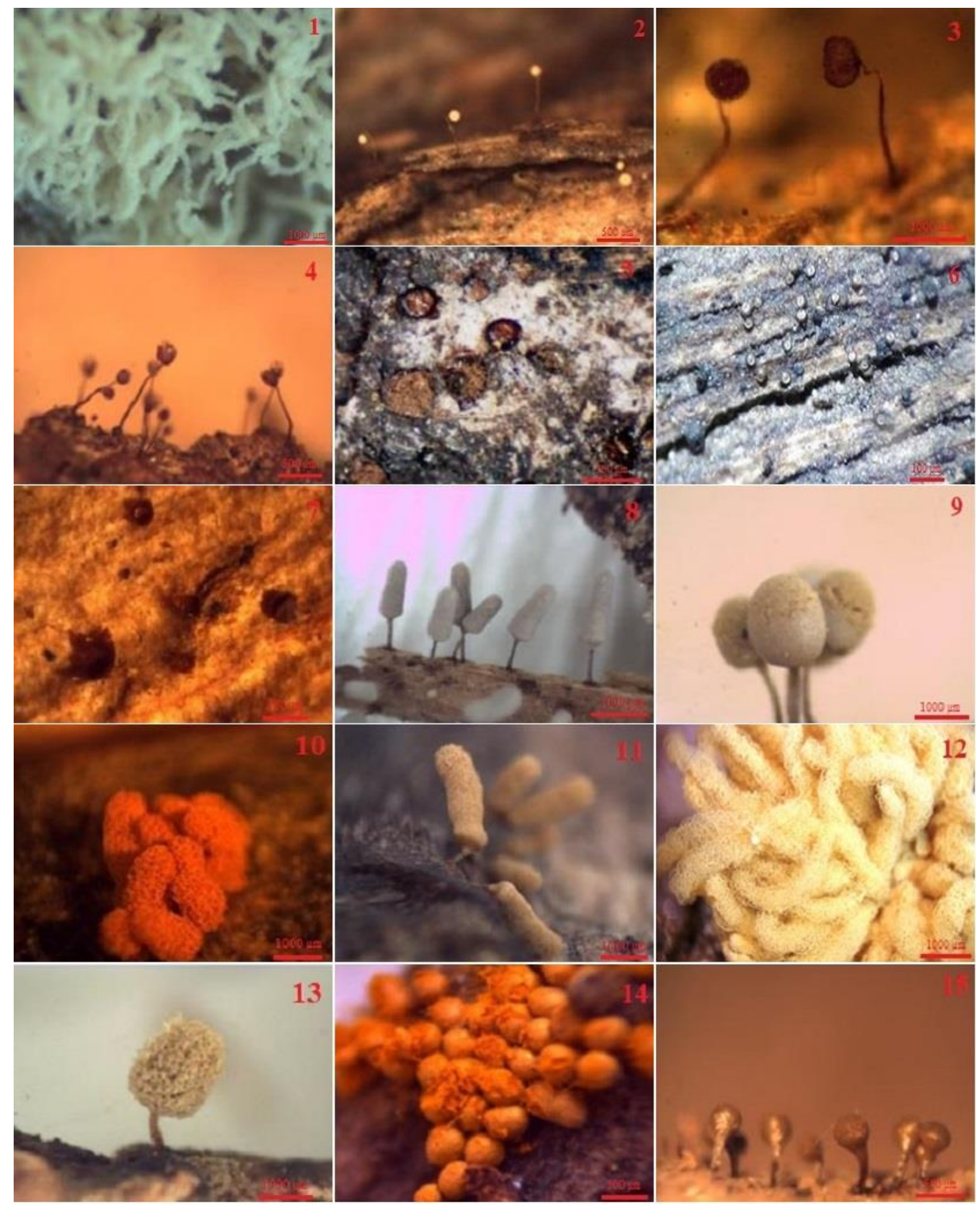

Figure 2. Myxomycetes images (1- Ceratiomyxa fruticulosa, 2- Echinostelium minutum, 3Cribraria cancellata, 4- C. violacea, 5- Licea castanea, 6- L. kleistobolus, 7- L. minima, 8Arcyria cinerea, 9- A. globosa, 10- A. incarnata, 11- A. minuta, 12- A. obvelata, 13- A. pomiformis, 14- Hemitrichia sp., 15- Trichia decipiens) 


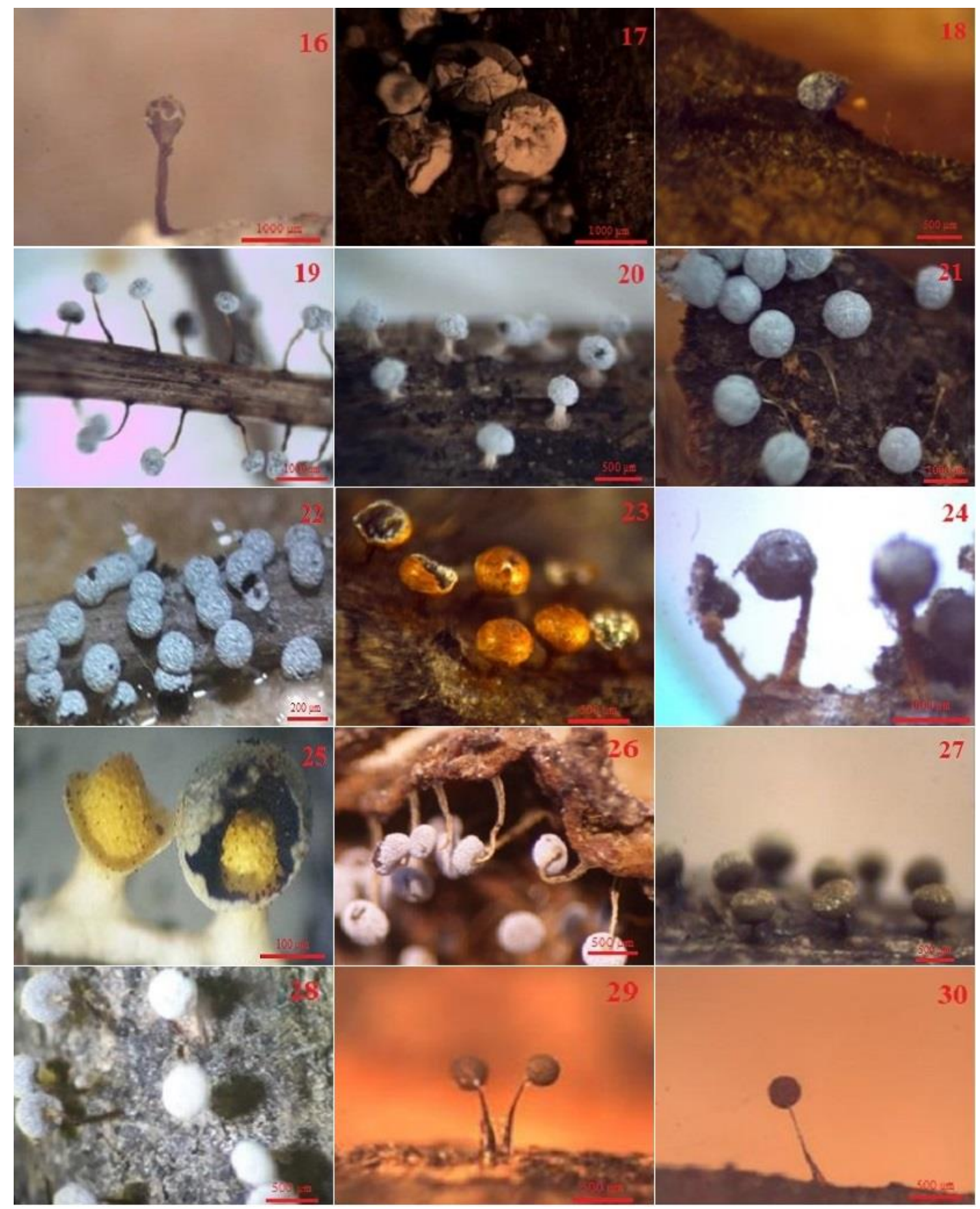

Figure 3. Myxomycetes images (Cont.) (16- T. munda, 17- Diderma hemisphaericum, 18Didymium clavus, 19- D. megalosporum, 20- D. squamulosum, 21- Badhamia dubia, 22- B. panicea, 23- Craterium sp., 24- C. dictyosporum, 25- Physarum sp., 26- P. album, $27-P$. oblatum, 28- P. robustum, 29- Collaria lurida, 30- Comatricha ellae) 


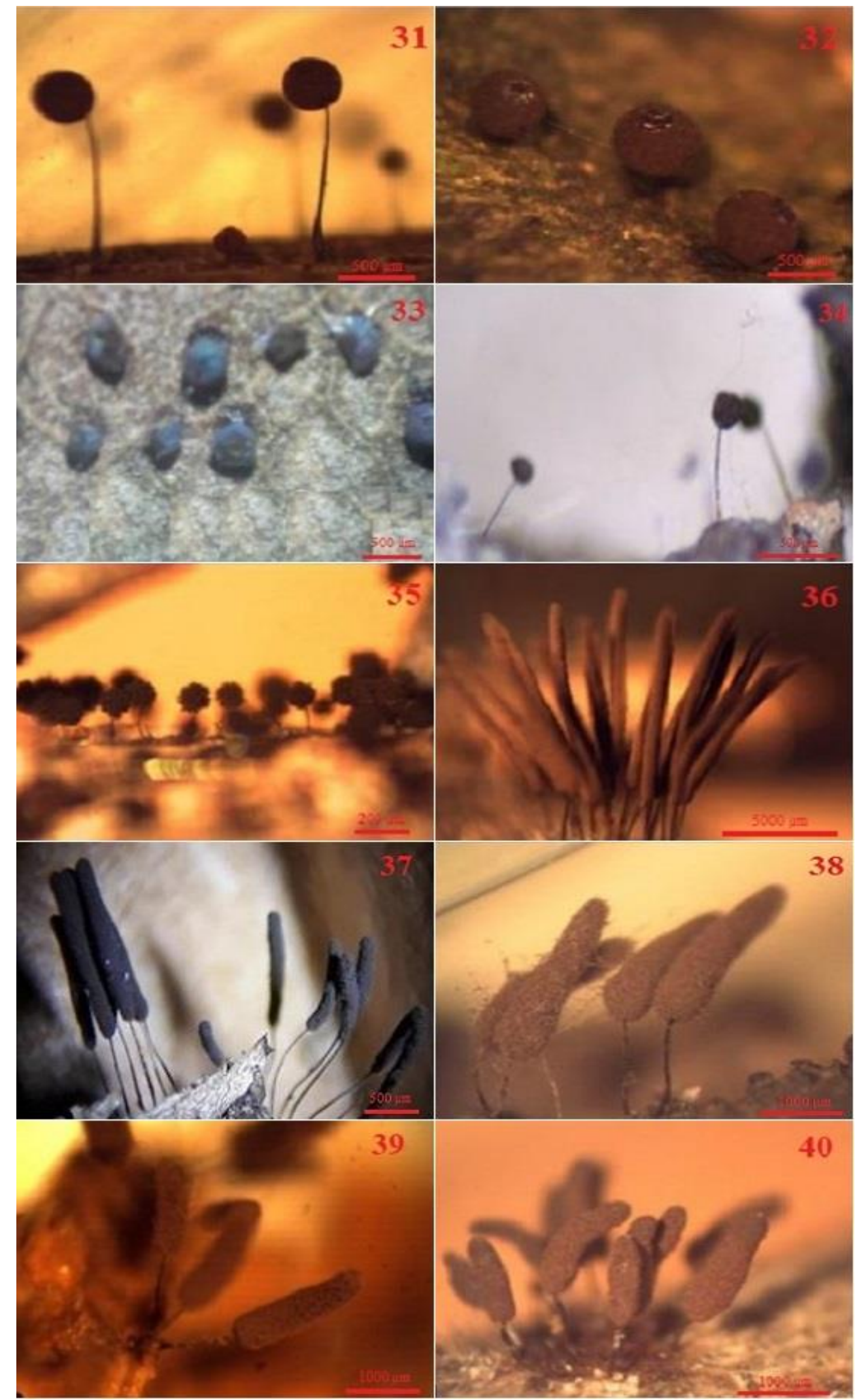

Figure 4. Myxomycete images (Cont.) (31- Comatricha nigra, 32- Enerthenema papillatum, 33Lamproderma arcyrioides, 34- Macbrideola cornea, 35- M. decapillata, 36- Stemonitis fusca, 37- Stemonitopsis amoena, 38- S. hyperopta, 39- S. reticulata, 40- S. subcaespitosa) 


\section{Discussion}

Myxomycetes spread on decayed Gymnosperm woods, leaves and debris. The Liceales, Trichiales and Stemonitales members are generally known to spread in coniferous forests (Martin and Alexopoulos, 1969; Stephenson \& Stempen, 1994; Ergül and Akgül, 2011; Baba et al., 2016). In the present study, myxomycete sporophores were collected from Pinus sp. and Quercus sp., leaves and coarse wood debris. Corticolous myxomycetes and lignicolous myxomycetes material were collected only from bark and rotten wood in the study area.

The distribution of the species determined in the study area included Stemonitidaceae 12, Physaraceae 8, Arcyriaceae 6, Didymiaceae 4, Trichiaceae 3, Liceaceae 3, Cribrariaceae 2, Ceratiomyxidae 1 and Echinosteliaceae 1 species.

Previous studies reported that Echinostelium minutum, Arcyria cinerea and Stemonitis fusca could grow on all types of substrates and in almost all parts of the world (Stephenson \& Stempen, 1994). These species were also identified in our study area.

Furthermore, A. pomiformis, A. cinerea, $C$. ellae and $C$. nigra were determined at several locations in the present study area. The detection of these common species was consistent with other studies conducted in Turkey (Ocak \& Hasenekoğlu, 2003; Yağız \& Afyon, 2007; Baba, 2015; Ergül et al., 2016).

\section{Conclusion}

In the present study, 40 species in 9 families and 19 genera were determined in order to determine myxomycetes diversity in Belen district. Also, the present study contributed to Turkish Myxobiota.

\section{Acknowledgements}

This study was prepared from a thesis study on Myxomycota (Myxomycota), which is distributed in the district of Belen (Hatay). We would like to thank the MKU Scientific Research Projects Commission for supporting this study. Project No: 8942

\section{References}

Alkan, S., Eroğlu, G. \& Kaşık, G. (2016). A new Myxomycete record from Turkey. Biological Diversity and Conservation, 9(2), 128-130.

Anonymous, 2019. https://belen.bel.tr

Baba, H., Gelen, M. \& Zümre, M. (2013). A new Myxomycetes record for Physarum Genus From Turkey. Biological Diversity and Conservation, 6, 49-51.

Baba, H. (2015). Investigation of Myxomycetes diversity on Kuseyr Mountain ; Three new records in Hatay/Turkey. Fresen. Environ. Bull. 24(11c), 4077-4086.

Baba, H. \& Zümre, M. (2015). A new Myxomycetes record from Turkey. Journal of Biology, Agriculture and Healthcare, 5(9), 14-16.

Baba, H., Zümre, M. \& Özyiğit, İ. (2016). A comparative biogeographical study of myxomycetes in four different habitats of eastern mediterranean part of Turkey, Fresen. Environ. Bull., 25(5), 1448-1459.

Baba, H. \& Arslan, Ç. (2017). Licea pescadorensis, A new Myxomycetes record for Turkey. Ĭgdır Univ. J. Inst. Sci. \& Tech., 7(4), 33-36.

Baba, H. \& Özyiğit, İ. (2017). Three new rare Myxomycetes (Myxogastrea) records from Hatay, Turkey. Fresen. Environ. Bull., 26(8), 4907-4910.

Baba, H. \& Er, A. (2018). Craterium dictyosporum : A new record of Myxomycetes from Hatay, Turkey. Acta Biologica Turcica, 31(1), 33-35

Baba, H. \& Doğan, Y. (2018). Investigation of Myxomycetes (Myxomycota) in South Amanos Mountains (Hatay-Turkey), Celal Bayar University Journal of Science, 14(3), 277-284

Baba, H., Gelen, M. \& Sevindik, M. (2018). Taxonomic investigation of myxomycetes in Altınözü, Turkey, Mycopath, 16(1), 23-31.

Baba, H. \& Sevindik, M. (2019). Mycetozoa of Turkey (checklist). Mycopath, 17(1), 1-14.

Dülger, B., Yıldız, I. \& Karabacak, E. (2016). A new myxomycetes record for the myxobiota of Turkey: Physarum melleum. International Journal of Botany Studies, 1(4), 37-38.

Ergül, C.C \& Akgül, H. (2011). Myxomycete diversity of Uludağ national park, Turkey. Mycotaxon, 116, 479.

Ergül, C.C., Akgül, H. \& Oran, R.B. (2016). New records of Myxogastrea taxa from Turkey. Oxidation Communications, 39(2), 1615-1623

Ergül, C.C., Dulger, B. \& Akgul, H. (2005a). Myxomycetes of Mezit stream valley of Turkey. Mycotaxon, 92, 239-242 
Ergül, C.C., Dulger, B., Oran, R.B. \& Akgul, H. (2005b). Myxomycetes of the western Black Sea Region of Turkey. Mycotaxon, 93, 362

Farr, M.L. (1981). True Slime Molds. Dubuque Iowa: Wm. C. Brown Comp.

Gilbert, H.C. \& Martin, G.W. (1933). Myxomycetes found on the bark of living trees. University of Iowa, Iowa Stud. Nat. Hist., 15 (3), 3-5.

Harkönen, M. \& Uotila, P. (1983). Turkish Myxomycetes developed in Moist Chamber Cultures, Karstenia, 23, 1-9.

Härkönen, M. \& Ukkola, T. (2000). Conclusions on myxomycetes compiled of twenty-five years from 4793 moist chamber cultures. Stapfia, 73, 105-112.

Ing, B. (1999). The Myxomycetes of Britain and Ireland. The Richmond Publishing Co.,Slough, England.

Lado, C. \& Pando, F. (1997). Flora Mycologica Iberica, 2. Madrid, Spain: CSIC.

Lado, C. (2019). An on line nomenclatural information system of EuMyxogastrea. Real Jardín Botánico, CSIC. Madrid, Spain. Last updated March 01, 2019. https://eumycetozoa.com

Martin, G.W. \& Alexopoulos, C.J. (1969). The Myxomycetes. 560, Iowa: University of Iowa press.

Martin, G.W., Alexopoulos, C.J. \& Farr, M.L. (1983). The Genera of Myxomycetes. Iowa City: University of Iowa press.
Neubert, H., Nowotny, W. \& Baumann, K. (1993). Die Myxomyceten (Band I). Gomaringen: Karlheinz Baumann Verlag

Neubert, H., Nowotny, W. \& Baumann, K. (1995). Die Myxomyceten (Band II). Karlheinz Baumann Verlag

Neubert, H., Nowotny, W., Baumann, K. \& Marx, H. (2000). Die Myxomyceten (Band III). Karlheinz Baumann Verlag

Ocak, İ. \& Hasenekoğlu, I. (2003). Myxomycetes from Erzurum, Bayburt and Gümüşhane Provinces. Turk J Bot., 27, 223-226.

Ocak, İ. \& Konuk, M. (2018). Diversity and ecology of Myxomycetes from Kütahya and Konya (Turkey) with four new records. Mycobiology, 46(3), 215-223.

Sesli, E., Akata, I., Denchev, T.T. \& Denchev, C.M. (2016). Myxomycetes in Turkey a checklist. Mycobiota, 6, 1-20.

Stephenson, S.L. \& Stempen, H. (1994). Myxomycetes: A Handbook of Slime Molds. Portland, USA: TimberPress.

Süerdem, T.B., Karabacak, E. \& Dülger, B. (2015). A new record of Diderma (Myxomycetes) from Turkey Mycologia Iranica, 2(2), 135-138.

Thind, K.S. (1977). The Myxomycetes of India. New Delhi: I.C.A.R.

Yağı, D. \& Afyon, A. (2007). The ecology and chorology of Myxomycetes in Turkey. Mycotaxon, 101, 279-282. 